\title{
$k$-cycle free one-factorizations of complete graphs
}

\author{
Mariusz Meszka \\ Faculty of Applied Mathematics, AGH University of Science and Technology, \\ Al. Mickiewicza 30, 30-059 Kraków, Poland \\ meszkaagh.edu.pl \\ Submitted: Dec 5, 2007; Accepted: Dec 10, 2008; Published: Jan 7, 2009 \\ Mathematics Subject Classifications: 05C70
}

\begin{abstract}
It is proved that for every $n \geq 3$ and every even $k \geq 4$, where $k \neq 2 n$, there exists one-factorization of the complete graph $K_{2 n}$ such that any two one-factors do not induce a graph with a cycle of length $k$ as a component. Moreover, some infinite classes of one-factorizations, in which lengths of cycles induced by any two one-factors satisfy a given lower bound, are constructed.
\end{abstract}

\section{Introduction}

A one-factor of a graph $G$ is a regular spanning subgraph of degree one. A one-factorization of $G$ is a set $F=\left\{F_{1}, F_{2}, \ldots, F_{n}\right\}$ of edge-disjoint one-factors such that $E(G)=$ $\bigcup_{i=1}^{n} E\left(F_{i}\right)$. Evidently, the union of two edge-disjoint one-factors is a two-factor consisting of cycles of even lengths.

The exact number $N(2 n)$ of all pairwise non-isomorphic one-factorizations of the complete graph $K_{2 n}$ is known only for $2 n \leq 14$; namely $N(4)=N(6)=1, N(8)=6, N(10)=$ 396, cf. [14], $N(12)=526,915,620$ [8], and $N(14)=1,132,835,421,602,062,347$ [10]. Moreover, Cameron [4] proved that $\ln N(2 n) \sim 2 n^{2} \ln (2 n)$ for sufficiently large $n$. Therefore, any investigations (including enumeration) regarding all one-factorizations of $K_{2 n}$ are deemed reasonable if they are restricted to a subclass which satisfies some additional properties. One of the obvious requirements concerns an isomorphism of graphs induced by pairs of one-factors. In this way, a question arises regarding the existence of uniform (perfect) one-factorizations. A one-factorization is uniform when the union of any two one-factors is isomorphic to the same graph $H$. In particular, if $H$ is connected (i.e. a Hamiltonian cycle), then a one-factorization is called perfect.

Perfect one-factorizations of complete graphs were introduced by Kotzig [11] and in known notation by Anderson [2]. Only three infinite classes of perfect one-factorizations are known, namely when $2 n-1$ is prime [11,3] and when $n$ is prime [1]. All other known examples of perfect one-factorizations of $K_{2 n}$ have been found using various methods, 
cf. [16, 17]. Perfect one-factorization conjecture, which claims the existence of perfect one-factorizations for every even order of the complete graph, is far from proven. Perfect one-factorizations are very rare among all one-factorizations; this argument is supported by a comparison of known numbers, $P(2 n)$, of all perfect pairwise non-isomorphic onefactorizations of $K_{2 n}$, with $N(2 n)$. There are $P(4)=P(6)=P(8)=P(10)=1, P(12)=$ 5, cf. [16, 17], $P(14)=23$ [7] and $P(16) \geq 88$ [15]. Uniform one-factorizations other than those which are perfect have been investigated far less, cf. [5, 14]. In fact, there are only three known infinite classes and several sporadic examples of uniform non-perfect one-factorizations.

In this context, weaker properties regarding lengths of cycles which are required to exist, or which are forbidden in the union of any two one-factors, may be considered. A one-factorization $F=\left\{F_{1}, F_{2}, \ldots, F_{n}\right\}$ of $G$ is said to be $k$-cycle free if the union of any two one-factors does not include the cycle $C_{k}$. Consequently, $F$ is $S$-cycle free if the union of any two one-factors does not include cycles of lengths from the set $S$. In particular, if $S=\{4,6, \ldots, k\}$, then $F$ is called $k^{<}$-cycle free. It can be said that $F$ has a cycle of length $k$ if there are two one-factors in $F$, the union of which includes $C_{k}$.

The aim of this paper is to find, for each $n$ and each even $k \geq 4$ such that $2 n \neq k$, a $k$-cycle free one-factorization of $K_{2 n}$. For $2 n \neq p+1$, where $p$ is a prime, or $2 n \neq \equiv 6,12,18$ $(\bmod 24)$, the existence of $2 n$-cycle free one-factorizations of $K_{2 n}$ is proven. Moreover, some infinite classes of $k^{<}$-cycle free one-factorizations are constructed.

\section{Constructions and their properties}

The following two facts are easily observed.

Claim 1 For $n \geq 2$, if $l$ is the minimum positive integer such that $\operatorname{gcd}(l, n)>1$, then $\operatorname{gcd}(l, n)=l$. Moreover, for odd $n^{\prime} \geq 3$, if $l^{\prime}$ is the minimum even positive integer such that $\operatorname{gcd}\left(l^{\prime}, n^{\prime}\right)>1$, then $\operatorname{gcd}\left(l^{\prime}, n^{\prime}\right)=l^{\prime} / 2$.

Claim 2 If $k>2 n \geq 4$, then any one-factorization of $K_{2 n}$ is $k$-cycle free.

The well-known canonical one-factorization $G K_{2 n}$ of $K_{2 n}$ has been published in Lucas' [13] and attributed to Walecki.

Construction A Let $V=\{\infty, 0,1, \ldots, 2 n-2\}$. Let $G K_{2 n}$ denote a one-factorization of $K_{2 n}$ which consists of one-factors $F_{i}=\{\{i-j, i+j\}: j=1,2, \ldots n-1\} \cup\{\infty, i\}$, for $i=0,1, \ldots 2 n-2$, where labels are taken modulo $2 n-1$.

It is well-known that $G K_{2 n}$ is perfect if and only if $2 n-1$ is prime [11]. Dinitz et. al. [6] investigated lengths of cycles which may appear in the union of any two one-factors in $G K_{2 n}$. Lemma 3 is a corollary to that result; a short proof is presented here in order to provide detailed constructions of cycles applied in further results.

Lemma 3 (cf. [6]) For $n \geq 3$ and even $k$ such that $4 \leq k \leq 2 n$, the one-factorization $G_{2 n}$ of $K_{2 n}$ contains a cycle of length $k$ if and only if $k / 2 \mid 2 n-1$ or $k-1 \mid 2 n-1$. 
Proof: Let $p=2 n-1$. Assume that $G K_{2 n}$ contains a cycle $C_{k}$ of length $k$ which appears in the union $H$ of two one-factors $F_{h}$ and $F_{i}$, where $h<i$ and $h, i \in\{0,1, \ldots, p-1\}$. Let $z=i-h$. Consider separately two cases.

Case I: $C_{k}$ contains the vertex $\infty$. Then neighbors of $\infty$ in $H$ are $h$ and $i$. Consecutive vertices along the cycle $C_{k}$ in $H$ are: $\infty, i, h-z, i+2 z, h-3 z, i+4 z, h-5 z, \ldots, i+(k-2) z$, $\infty$, where $\mathrm{k}$ is the minimum even positive integer such that $i+(k-2) z \equiv h \quad(\bmod p)$ (which is equivalent to $(k-1) z \equiv 0 \quad(\bmod p))$. Since $0<z<p, \operatorname{gcd}(k-1, p)=k-1$ follows by Claim 1 .

Case II: $C_{k}$ does not contain $\infty$. Let $h+x$ be a vertex of $C_{k}$. Then $x \neq 0$ and neighbors of $h+x$ in $H$ are $h-x$ and $i+z-x$. Consecutive vertices along $C_{k}$ in $H$ are: $h+x$, $h-x, i+z+x, h-2 z-x, i+3 z+x, h-4 z-x, \ldots, h-(k-2) z-x, h+x$, where $k$ is the minimum even positive integer such that $h-(k-2) z-x \equiv i+z-x \quad(\bmod p)$. Similarly to the above, since $0<z<p$, by the equivalence $k z \equiv 0 \quad(\bmod p)$ and Claim $1, \operatorname{gcd}(k, p)=k / 2$.

To prove sufficiency, suppose first that $k \leq 2 n$ and $k / 2 \mid p$. Then $k \equiv 2(\bmod 4)$. In order to find a cycle of length $k$, take two one-factors $F_{0}$ and $F_{i}$, where $i=\frac{p}{k / 2}$. Let $l$ be the length of a cycle which does not contain $\infty$ in the union of $F_{0}$ and $F_{i}$. Then, repeating calculations of Case II, $l$ is the minimum even positive integer such that $l i \equiv 0$ $(\bmod p)$. Then $\frac{l p}{k / 2} \equiv 0 \quad(\bmod p)$ and next, since $k / 2$ is odd, $l=k$. Similarly, for any even $k \leq 2 n$ where $k-1 \mid p$, two one-factors $F_{0}$ and $F_{j}$ are taken, where $j=\frac{p}{k-1}$. If $l$ is the length of a cycle which contains $\infty$, then as in Case I, $l$ is the minimum even positive integer such that $(l-1) j \equiv 0 \quad(\bmod p)$. Thus $l=k$.

The above Lemma 3 is equivalent to the following result.

Corollary 4 For $n \geq 3$ and even $k \geq 4$, the one-factorization $G K_{2 n}$ of $K_{2 n}$ is $k$-cycle free if and only if $k / 2 \nmid 2 n-1$ and $k-1 \nmid 2 n-1$.

By Lemma 3, the one-factorization $G K_{2 n}$ has a trivial lower bound on the minimum length of cycles it contains.

Corollary 5 Let $r$ be the minimum prime factor of $2 n-1$. If $r \geq 5$, then the onefactorization $G K_{2 n}$ of $K_{2 n}$ is $(r-1)^{<}$-cycle free.

Lemma 3 immediately yields another property of $G K_{2 n}$. Namely, for any order $2 n$, $G K_{2 n}$ cannot be a non-perfect uniform one-factorization because $G K_{2 n}$ contains a cycle of length $2 n$.

Another well-known one-factorization of the complete graph of order $2 n$ for odd $n$ is denoted by $G A_{2 n}[2]$.

Construction $\mathbf{B}$ Let $n$ be odd. In what follows, labels of vertices are taken modulo $n$. Let $V=V_{0} \cup V_{1}$, where $V_{m}=\left\{0_{m}, 1_{m}, \ldots,(n-1)_{m}\right\}$ for $m=0,1$. Let $G A_{2 n}$ be a one-factorization of $K_{2 n}$ with one-factors $F_{0}, F_{1}, \ldots, F_{2 n-2}$. Let $F_{i}=\left\{\left\{(i-j)_{m},(i+\right.\right.$ $\left.\left.j)_{m}\right\}: j=1,2, \ldots(n-1) / 2, m=0,1\right\} \cup\left\{i_{0}, i_{1}\right\}$, for $i=0,1, \ldots n-1$. Moreover, let $F_{n+i}=\left\{\left\{j_{0},(j+i+1)_{1}\right\}: j=0,1, \ldots n-1\right\}$, for $i=0,1, \ldots n-2$. 
It is well-known that $G A_{2 n}$ is perfect if and only if $n$ is prime [1]. The following presents a stronger property of $G A_{2 n}$.

Lemma 6 For odd $n \geq 3$ and even $k$ such that $4 \leq k \leq 2 n$, the one-factorization $G A_{2 n}$ of $K_{2 n}$ contains a cycle of length $k$ if and only if $k / 2 \mid n$.

Proof: Assume first that $G A_{2 n}$ contains a cycle $C_{k}$ which is included in the union $H$ of two one-factors $F_{h}$ and $F_{i}$, where $h<i$ and $h, i \in\{0,1, \ldots, 2 n-2\}$. Consider separately three cases.

Case I: $h<i \leq n-1$. Note that, if in the construction of $G K_{n+1}$ the vertex subset $V\left(K_{n+1}\right) \backslash\{\infty\}$ is replaced with $V_{m}$, for $m=0,1$, and moreover, $G K_{n+1}$ is restricted to the vertices of $V_{m}$, then a near one-factorization of $K_{n}$ into near one-factors $F_{i}^{m}=$ $\left\{\left\{(i-j)_{m},(i+j)_{m}\right\}: j=1,2, \ldots(n-1) / 2\right\}$ is obtained, where $i=0,1, \ldots, n-1$. It is clear that $F_{i}^{m} \subset F_{i}$ (the one-factor of $G A_{2 n}$ ) for every admissible $i$ and $m$. A cycle $C_{k}$ in $H$ has all vertices either in $V_{0}$ or in $V_{1}$ or in both subsets together. In the previous two cases $C_{k}$ corresponds to a cycle of the same length either in $F_{h}^{0} \cup F_{i}^{0}$ or in $F_{h}^{1} \cup F_{i}{ }^{1}$. Then, by Case II in the proof of Lemma $3, \operatorname{gcd}(k, n)=k / 2$. In the latter case, $k \equiv 2 \quad(\bmod 4)$ and $C_{k}$ consists of two paths of length $k / 2-1$ (one of them with all vertices in $V_{0}$ and the other one with all vertices in $V_{1}$ ) joint together by the edges $\left\{h_{0}, h_{1}\right\}$ (of $F_{h}$ ) and $\left\{i_{0}, i_{1}\right\}$ ( of $F_{i}$ ). These two paths correspond to a path with endvertices $h$ and $i$ included in a cycle of length $k / 2+1$ ( which contains the vertex $\infty$ ), induced by one-factors with indices $h$ and $i$ in $G K_{n+1}$. Thus, by Case I in the proof of Lemma $3, \operatorname{gcd}(k / 2, n)=k / 2$ holds.

Case II: $h<n \leq i$. Consider two subcases.

II.A: $h_{0}$ is not a vertex of the cycle $C_{k}$ in $H$. Then also $h_{1}$ is not in $C_{k}$. Note that the length of $C_{k}$ is divisible by 4 . Let $(h+x)_{0}$ be a vertex of $C_{k}$ for some $x \neq 0$. Then neighbors of $(h+x)_{0}$ in $H$ are $(h-x)_{0}$ and $(h+x+i+1)_{1}$. Consecutive vertices along the cycle $C_{k}$ in $H$ are: $(h+x)_{0},(h+x+i+1)_{1},(h-x-i-1)_{1},(h-x-2 i-2)_{0}$, $(h+x+2 i+2)_{0},(h+x+3 i+3)_{1},(h-x-3 i-3)_{1}, \ldots,\left(h-x-\frac{k(i+1)}{2}\right)_{0},(h+x)_{0}$, where $k$ is the minimum even positive integer such that $h-x-\frac{k(i+1)}{2} \equiv h-x \quad(\bmod n)$. Since $n<i+1<2 n$, by the above equivalence $\frac{k}{2}(i+1) \equiv 0(\bmod n)$ and Claim 1 , $\operatorname{gcd}(k / 2, n)=k / 2$.

II.B: $h_{0}$ is a vertex of $C_{k}$. Then $h_{1}$ is in $C_{k}$ as well. Note that $k \equiv 2(\bmod 4)$. The neighbors of $h_{0}$ in $H$ are $h_{1}$ and $(h+i+1)_{1}$. Consecutive vertices along the cycle $C_{k}$ in $H$ are: $h_{0},(h+i+1)_{1},(h-i-1)_{1},(h-2 i-2)_{0},(h+2 i+2)_{0},(h+3 i+3)_{1}$, $(h-3 i-3)_{1}, \ldots,\left(h+\frac{k(i+1)}{2}\right)_{1}, h_{0}$, where $k$ is the minimum even positive integer such that $h+\frac{k(i+1)}{2} \equiv h \quad(\bmod n)$. Analogously to the previous case, since $n<i+1<2 n$, by Claim $1, \operatorname{gcd}(k / 2, n)=k / 2$ is easily observed.

Case III: $n \leq h<i$. Then neighbors of $y_{0}$ in $H$ are $(y+h+1)_{1}$ and $(y+i+1)_{1}$. Consecutive vertices along $C_{k}$ in $H$ are: $y_{0},(y+i+1)_{1},(y+i-h)_{0},(y+2 i-h+1)_{1}$, $(y+2 i-2 h)_{0}, \ldots,\left(y+\frac{k i-(k-2) h+2}{2}\right)_{1}, y_{0}$, where $y+\frac{k i-(k-2) h+2}{2} \equiv y+h+1 \quad(\bmod n)$. Similarly to the previous case, since $0<i-h<n$, by $\frac{k}{2}(i-h) \equiv 0 \quad(\bmod n)$ and Claim $1, \operatorname{gcd}(k / 2, n)=k / 2$ holds.

To show sufficiency, suppose that $k \leq 2 n$ and $k / 2 \mid n$. To find a cycle of length $k$, take one-factors $F_{n}$ and $F_{i}$ such that $i=n+\frac{n}{k / 2}$. Note that, if $l$ is the length of a cycle in the 
union of $F_{n}$ and $F_{i}$, then $l$ is the minimum even positive integer such that $\frac{l}{2}(i-n) \equiv 0$ $(\bmod n)\left(\right.$ cf. calculations of Case III above). Thus $\frac{l}{2} \frac{n}{k / 2} \equiv 0 \quad(\bmod n)$, whence $l=k$.

Lemma 6 is equivalent to the following.

Corollary 7 For odd $n \geq 3$ and even $k \geq 4$, the one-factorization $G A_{2 n}$ of $K_{2 n}$ is $k$-cycle free if and only if $k / 2 \nmid n$.

Lemma 6 immediately provides a lower bound on the minimum length of cycles in $G A_{2 n}$.

Corollary 8 Let $n$ be odd and $n \geq 3$. Let $r$ be the minimum prime factor of $n$. Then the one-factorization $G A_{2 n}$ of $K_{2 n}$ is $(2 r-2)^{<}$-cycle free.

Lemma 6 also yields an obvious corollary that $G A_{2 n}$ cannot be a non-perfect uniform one-factorization.

Presented below is an inductive construction for another family of one-factorizations of $K_{2 n}$.

Construction $\mathbf{C}$ Let $n$ be even. In what follows, labels of vertices are taken modulo $n$. Let $V=V_{0} \cup V_{1}$, where $V_{m}=\left\{0_{m}, 1_{m}, \ldots,(n-1)_{m}\right\}$ for $m=0,1$. Let $\bar{F}=\left\{\bar{F}_{0}, \bar{F}_{1}, \ldots, \bar{F}_{n-2}\right\}$ be a $k$-cycle free one-factorization of $K_{n}$, where $\bar{V}=V\left(K_{n}\right)=$ $\{0,1, \ldots, n\}$. Two copies of $\bar{F}$ are taken by replacing $\bar{V}$ with $V_{0}$ and $V_{1}$, respectively. In this way, $n-1$ one-factors $F_{i}$ of $K_{2 n}$ are obtained, $i=0,1, \ldots, n-2$. The $n$th one-factor is $F_{n-1}=\left\{\left\{j_{0}, j_{1}\right\}: j=0,1, \ldots n-1\right\}$. Remaining $n-1$ one-factors are built based on onefactors in $\bar{F}$; namely, if $\left\{v_{0}, u_{0}\right\}$ is the edge of one-factor $\bar{F}_{h}$, for some $h \in\{0,1, \ldots, n-2\}$, then $\left\{v_{0}, u_{1}\right\}$ and $\left\{v_{1}, u_{0}\right\}$ are the edges of one-factor $F_{n+h}$.

The above method allows for the construction of $k$-cycle free one-factorizations of $K_{2 n}$, where $n$ is even and $k \not \equiv 4(\bmod 8)$.

Lemma 9 For even $n \geq 4$ and even $k \geq 6$ such that $k \not \equiv 4(\bmod 8)$, if there is a $k$-cycle free one-factorization of $K_{n}$, then a $k$-cycle free one-factorization of $K_{2 n}$ exists.

Proof: Assume that a k-cycle free one-factorization $\bar{F}$ of $K_{n}$ is given. Let $H$ be the union of two one-factors $F_{h}$ and $F_{i}$ in the one-factorization of $K_{2 n}$ obtained by applying Construction $\mathrm{C}$, where $h<i$ and $h, i \in\{0,1, \ldots, 2 n-2\}$. If both $h, i<n-1$, then $H$ does not contain $C_{k}$ because all cycles in $H$ are, in fact, copies of cycles in the given one-factorization $\bar{F}$ of $K_{n}$ which is k-cycle free. If $i=n-1$ or $h=n-1$, one can see that every cycle in $H$ has length 4 . In what follows, assume that $i \geq n$. If $i-$ $h=n$, it is evident that every cycle in $H$ has length 4 as well. Otherwise $i-h \neq n$. Note that every cycle in $H$ corresponds to a cycle in the union of one-factors $\bar{F}_{h}$ and $\bar{F}_{i-n}$ in $K_{n}$. Let $C_{l}$ denote a cycle of length $l$ in $\bar{F}_{h} \cup \bar{F}_{i-n}$ with consecutive vertices $v^{1}, v^{2}, v^{3}, \ldots, v^{l}$. Suppose that $h<n-1$. Note that $C_{l}$ corresponds either to a cycle $C_{l}^{\prime}($ if $l \equiv 0 \quad(\bmod 4))$ of length $l$ or to a cycle $C_{2 l}^{\prime \prime}($ if $l \equiv 2 \quad(\bmod 4))$ of length $2 l$ in 
$H$; consecutive vertices of $C_{l}^{\prime}$ are $v_{0}^{1}, v_{0}^{2}, v_{1}^{3}, v_{1}^{4}, v_{0}^{5}, v_{0}^{6}, \ldots, v_{1}^{l-1}, v_{1}^{l}$, while $C_{2 l}^{\prime}$ has vertices $v_{0}^{1}, v_{0}^{2}, v_{1}^{3}, v_{1}^{4}, \ldots, v_{0}^{l-1}, v_{0}^{l}, v_{1}^{l+1}, v_{1}^{l+2}, \ldots, v_{1}^{2 l-1}, v_{1}^{2 l}$. In the latter case, by the assumption, $k \neq 2 l$. Consider the last case $n \leq h$. Then $C_{l}$ corresponds to a cycle $C_{l}^{\prime \prime \prime}$ of the same length $l$ in $H$ with consecutive vertices $v_{0}^{1}, v_{1}^{2}, v_{0}^{3}, v_{1}^{4}, \ldots, v_{0}^{l-1}, v_{1}^{l}$. Hence, since $K_{n}$ is $k$-cycle free, $k \neq l$ and the assertion holds.

By the above Lemma 9 , if $2 n \equiv 0 \quad(\bmod 8)$, then a one-factorization built by applying Construction $\mathrm{C}$ does not contain a cycle of length $2 n$. Moreover, starting from $n=4$ and applying the above inductive construction for consecutive powers of 2 , a well-known class of uniform one-factorizations of complete graphs with all cycles of length 4 is easily obtained, cf. [4].

Construction $\mathrm{C}$ also enables the building of a $\{k / 2, k\}$-cycle free one-factorization of $K_{2 n}$, using a given $\{k / 2, k\}$-cycle free one-factorization of $K_{n}$.

Lemma 10 For even $n \geq 4$ and even $k \geq 12$ such that $k \equiv 4(\bmod 8)$, if there is a $\{k / 2, k\}$-cycle free one-factorization of $K_{n}$, then a $\{k / 2, k\}$-cycle free one-factorization of $K_{2 n}$ exists.

Proof: The assertion follows immediately from the proof of Lemma 9. Namely, by the assumption, a given one-factorization of $K_{n}$ does not contain a cycle of length $l$ such that $l \equiv 2 \quad(\bmod 4)$. Hence, by the proof of Lemma 9 , every cycle in a one-factorization of $K_{2 n}$, obtained by applying Construction $\mathrm{C}$, has either length 4 or has the same length as a corresponding cycle in a given one-factorization of $K_{n}$.

The next infinite class of one-factorizations yields further examples of $k$-cycle free and $k^{<}$-cycle free one-factorizations of complete graphs.

Construction $\mathbf{D}$ Let $p \geq 3$ be a prime and $r=(p-1) / 2$. Let $n$ be an odd integer such that $n \geq p$ and $\operatorname{gcd}(n, r)=1$. Let $r^{-1}$ be the inverse of $r$ in $\mathbb{Z}_{n}$. In what follows, labels of vertices are taken modulo $n$, while indices are taken modulo $p$. Consider a onefactorization of $K_{p n+1}$ denoted by $H K_{p n+1}$. Let $V=V_{0} \cup V_{1} \cup \ldots \cup V_{p-1}$, where $V_{m}=$ $\left\{\infty, 0_{m}, 1_{m}, \ldots,(n-1)_{m}\right\}$ for $m=0,1, \ldots, p-1$. Thus $V_{0} \cap V_{1} \cap \ldots V_{p-1}=\{\infty\}$. Let $F_{m n+i}=\left\{\left\{(i-j)_{m},(i+j)_{m}\right\}: j=1,2, \ldots(n-1) / 2\right\} \cup\left\{i_{m}, \infty\right\} \cup\left\{\left\{j_{m-s},-(j+(i+\right.\right.$ $\left.\left.\left.m) r^{-1}\right)_{m+s}\right\}: j=0,1, \ldots n-1, s=1,2, \ldots, r\right\}$ for $i=0,1, \ldots n-1, m=0,1, \ldots, p-1$.

Note that $H K_{n p+1}$ is an extension of $G K_{p}$ : one-factorization induced by every $V_{i}$ is the one-factorization $G K_{n+1}$ of $K_{n+1}$. Moreover, if every set $V_{i} \backslash \infty$ is replaced by a single vertex $u_{i}$, and all edges with the same endvertices are contracted to a single edge, loops being removed, then the corresponding one-factorization $G K_{p+1}$ of $K_{p+1}$ would be obtained.

Presented below are investigations into possible lengths of cycles in $H K_{p n+1}$.

Lemma 11 For odd prime $p$ and for odd $n$ such that $n \geq p$ and $\operatorname{gcd}(n,(p-1) / 2)=1$, and for even $k$ such that $4 \leq k \leq p n+1$, the one-factorization $H K_{p n+1}$ of $K_{p n+1}$ contains a cycle of length $k$ if and only if one of the following conditions holds: 
(1) $k \leq n+1$ and $k-1 \mid n$,

(2) $k>n+1$ and $k-1 \mid n p$,

(3) $k \leq 2 n$ and $k / 2 \mid n$,

(4) $k>2 n$ and $k / 2 \mid n p$.

Proof: Assume that $H K_{p n+1}$ contains a cycle of length $k$ which appears in the union $H$ of one-factors $F_{h}$ and $F_{i}$, where $h<i$ and $h, i \in\{0,1, \ldots, p n-1\}$. Consider separately two cases.

Case I: $m n \leq h<i<(m+1) n$ for some $m \in\{0,1, \ldots, p-1\}$. One-factorization induced by $V_{m}$ is the one-factorization $G K_{n+1}$ of $K_{n+1}$ and therefore, by Lemma 3, either condition (1) or (3) is satisfied when $k \leq n+1$ and all vertices of $C_{k}$ come from $V_{m}$. Consider the case where all vertices of $C_{k}$ are in $V \backslash V_{m}$. In fact, all vertices of $C_{k}$ are in $V_{m-s} \cup V_{m+s}$ for some $s \in\{1,2 \ldots, r\}$. Then clearly $k \leq 2 n$. Let $y_{m-s}$ be a vertex of $C_{k}$. Neighbors of the vertex $y_{m-s}$ are $-\left(y+(h+m) r^{-1}\right)_{m+s}$ and $-\left(y+(i+m) r^{-1}\right)_{m+s}$. Consecutive vertices along the cycle $C_{k}$ are: $y_{m-s},-\left(y+(h+m) r^{-1}\right)_{m+s},\left(y+(h-i) r^{-1}\right)_{m-s},-\left(y+(2 h-i+m) r^{-1}\right)_{m+s}$, $\left(y+(2 h-2 i) r^{-1}\right)_{m-s}, \ldots,-\left(y+\frac{k h-(k-2) i+2 m}{2} r^{-1}\right)_{m+s}, y_{m-s}$, where $k$ is the minimum even positive integer such that $-y-\frac{k h-(k-2) i+2 m}{2} r^{-1} \equiv-y-(i+m) r^{-1} \quad(\bmod n)$. Since $0<i-h<n$, then $0<(i-h) r^{-1}<n$ and, by the equivalence $\frac{k}{2}(i-h) r^{-1} \equiv 0 \quad(\bmod n)$ and Claim 1, $\operatorname{gcd}\left(\frac{k}{2}, n\right)=\frac{k}{2}$ and then $(3)$ holds.

Case II: $m n \leq h<(m+1) n$ and $q n \leq i<(q+1) n$ for some $m, q \in\{0,1, \ldots, p-1\}$, $m<q$. Let $z=q-m$. Consider two subcases.

II.A: $\infty$ is a vertex of $C_{k}$. Then $k \equiv p+1 \quad(\bmod 2 p)$. Neighbors of $\infty$ in $H$ are $h_{m}$ and $i_{q}$. Note that indices of consecutive vertices in the cycle $C_{k}$ appear in the order according to the labels of vertices in Case I of the proof of Lemma 3 . Thus the first $p+1$ consecutive vertices along $C_{k}$ in $H$ are: $\infty, i_{q}=i_{m+z},-(h+r i+m) r_{m-z}^{-1},(h+(r-$ $1) i+m-q) r_{m+3 z}^{-1},-(2 h+(r-1) i+2 m-q) r_{m-3 z}^{-1},(2 h+(r-2) i+2 m-2 q) r_{m+5 z}^{-1}$, $\ldots,(r h+(r-r) i+r m-r q) r_{m+p z}^{-1}=(h-z)_{m}$. Note that $(h-z)_{m} \neq h_{m}$ because $0<z<p \leq n$. Thus the neighbor of $(h-z)_{m}$ in $F_{h}$ is $(h+z)_{m}$. Moreover, $(i+2 z)_{m+z} \neq$ $i_{m+z}$. Then the next $2 p$ consecutive vertices along $C_{k}$ in $H$ are: $(h+z)_{m}=(h+z)_{q-z}$, $-(r z+r h+i+q) r_{q+z}^{-1},(r z+(r-1) h+i+q-m) r_{q-3 z}^{-1},-(r z+(r-1) h+2 i+2 q-m) r_{q+3 z}^{-1}$, $(r z+(r-2) h+2 i+2 q-2 m) r_{q-5 z}^{-1}, \ldots,(r z+(r-r) h+r i+r q-r m) r_{q-p z}^{-1}=(i+$ $2 z)_{m+z},(i-2 z)_{m+z},-(-2 r z+h+r i+m) r_{m-z}^{-1},(-2 r z+h+(r-1) i+m-q) r_{m+3 z}^{-1}$, $-(-2 r z+2 h+(r-1) i+2 m-q) r_{m-3 z}^{-1},(-2 r z+2 h+(r-2) i+2 m-2 q) r_{m+5 z}^{-1}, \ldots$, $(-2 r z+r h+(r-r) i+r m-r q) r_{m+p z}^{-1}=(h-3 z)_{m}$. Therefore, after the next $\frac{k-(3 p+1)}{2 p}$ segments, each of which contains $2 p$ vertices, the $k$ th vertex in $C_{k}$ is $\left(h-\frac{k-1}{p} z\right)_{m}=h_{m}$. Since $0<z<p \leq n$, if $k$ is the minimum even positive integer such that $\frac{k-1}{p} z \equiv 0$ $(\bmod n)$, then $k-1>n$ and moreover, by Claim $1, \operatorname{gcd}\left(\frac{k-1}{p}, n\right)=\frac{k-1}{p}$. Thus $(2)$ is satisfied.

II.B: $\infty$ is not a vertex of $C_{k}$. Then $k \equiv 0 \quad(\bmod 2 p)$. Let $(h+x)_{m}$ be a vertex of $C_{k}$. Then $x \neq 0$ and neighbors of $(h+x)_{m}$ in $H$ are $(h-x)_{m}$ and $\left(-h+x-(i+q) r^{-1}\right)_{q+z}$. First segment of $2 p$ consecutive vertices along $C_{k}$ is (cf. second segment of $C_{k}$ in Subcase II.A): $(h+x)_{m}=(h+x)_{q-z},-(r x+r h+i+q) r_{q+z}^{-1},(r x+(r-1) h+i+q-m) r_{q-3 z}^{-1}$, 
$\ldots,(i+x+z)_{q}=(i+x+z)_{m+z},(i-x-z)_{m+z},-(-r x+h+r i+(r+1) m-r q) r_{m-z}^{-1}$, $(-r x+h+(r-1) i+(r+1) m-(r+1) q) r_{m+3 z}^{-1}, \ldots,(h-x-2 z)_{m} \neq(h-x)_{m}$. After the next $\frac{k}{2 p}-1$ segments, each of which contains $2 p$ vertices, we end up at $\left(h-x-\frac{k}{p} z\right)_{m}=(h-x)_{m}$. Since $0<z<p \leq n$ and moreover, $k$ is the minimum even positive integer such that $\frac{k}{p} z \equiv 0 \quad(\bmod n), k>2 n$ holds and, by Claim $1, \operatorname{gcd}\left(\frac{k}{p}, n\right)=\frac{k}{2 p}$. Hence (4) is satisfied.

To prove sufficiency, in order to find a cycle of length $k$, take the union of two onefactors $F_{0}$ and $F_{i}$. Let $i=\frac{n}{k-1}$ if $k \leq n+1$ and $k-1 \mid n$. Thus $1 \leq i<n$. Let $l$ be the length of a cycle in the union of $F_{0}$ and $F_{i}$ which contains $\infty$ and with all vertices in $V_{0}$. Then, by applying calculations of Case I in the proof of Lemma $3, l$ is the minimum even positive integer such that $(l-1) i \equiv 0 \quad(\bmod n)$. Thus $\frac{l-1}{k-1} n \equiv 0 \quad(\bmod n)$ and therefore $l=k$. Similarly, let $i=\frac{n r}{k / 2} \quad(\bmod n)$ if $k \leq 2 n$ and $k / 2 \mid n$. Hence, if $l$ is the length of a cycle in $F_{0} \cup F_{i}$ with all vertices in $V_{p-1} \cup V_{1}$, by calculations as in Case I above, $l$ is the minimum even positive integer such that $\frac{l}{2} i r^{-1} \equiv 0 \quad(\bmod n)$. Hence $l=k$. Analogously, let $i=n \frac{n p}{k-1}(\geq n)$ if $k>n+1 \geq p+1$ and $k-1 \mid n p$. If $l$ is the length of a cycle in $F_{0} \cup F_{i}$ which contains $\infty$, by calculations as in Subcase II.A above, $l$ is the minimum even positive integer such that $\frac{l-1}{p} z \equiv 0 \quad(\bmod n)$, where $z=i / n=\frac{n p}{k-1}<n$. Then $\frac{l-1}{p} \frac{n p}{k-1} \equiv 0 \quad(\bmod n)$, whence $k=l$. In the last case, if $k>2 n \geq 2 p$ and $k / 2 \mid n p$, then $i=n \frac{n p}{k / 2}>n$. Note that $k \equiv 2 \quad(\bmod 4)$. If $l$ is the length of a cycle in the union $F_{0} \cup F_{i}$ which does not contain $\infty$, then $l$ is the minimum even positive integer such that $\frac{l}{p} z \equiv 0$ $(\bmod n)$, where $z=i / n=\frac{n p}{k / 2}<n$, cf. Subcase II.B. Hence $\frac{l}{p} \frac{n p}{k / 2} \equiv 0 \quad(\bmod n)$ and, since $k / 2$ is odd, $k=l$ holds.

Lemma 11 is equivalent to the following result.

Corollary 12 For odd prime $p$ and for odd $n$ such that $n \geq p$ and $\operatorname{gcd}(n,(p-1) / 2)=1$, and for even $k \geq 4$, the one-factorization $H K_{p n+1}$ of $K_{p n+1}$ is $k$-cycle free if and only if all of the following conditions hold:

(1) $k-1 \nmid n$ if $k \leq n+1$,

(2) $k-1 \nmid n p$ if $k>n+1$,

(3) $k / 2 \nmid n$ if $k \leq 2 n$,

(4) $k / 2 \nmid n p$ if $k>2 n$.

Lemma 11 yields a trivial lower bound on the minimum length of cycles in $H K_{p n+1}$.

Corollary 13 Let $p$ be an odd prime and $n$ be odd such that $n \geq p$ and $\operatorname{gcd}(n,(p-1) / 2)=$ 1. Let $r$ be the minimum prime factor of $n$. If $r \geq 5$, then the one-factorization $H K_{p n+1}$ of $K_{p n+1}$ is $(r-1)^{<}$-cycle free.

It is clear that $H K_{p n+1}$ cannot be uniform. Taking two one-factors $F_{0}$ and $F_{1}$, its union $H$ has a cycle of length $n+1$ with all vertices in $V_{0}$, while one-factors $F_{0}$ and $F_{n}$ make a Hamiltonian cycle in $K_{p n+1}$.

The next inductive construction, similar to $H K_{p n+1}$, produces a one-factorization of $K_{p n+1}$ for odd $n$ and odd prime $p$, which does not have cycles of even lengths $k$, where $k \not \equiv 0, p+1 \quad(\bmod 2 p)$ or $k=p+1$. 
Construction $\mathbf{E}$ Let $p \geq 3$ be a prime and $r=(p-1) / 2$. Let $n$ be an odd integer such that $n \geq p$ and $\operatorname{gcd}(n, r)=1$. Let $r^{-1}$ be the inverse of $r$ in $\mathbb{Z}_{n}$. In what follows, labels of vertices are taken modulo $n$, while indices are taken modulo $p$. Let $V=V_{0} \cup V_{1} \cup \ldots \cup V_{p-1}$, where $V_{m}=\left\{\infty, 0_{m}, 1_{m}, \ldots,(n-1)_{m}\right\}$ for $m=0,1, \ldots, p-1$. Let $\tilde{F}$ be a $k$-cycle free onefactorization of $K_{n+1}$, where $\tilde{V}=V\left(K_{n+1}\right)=\{\infty, 0,1, \ldots, n-1\}$. Let $\tilde{F}_{i}$ be a one-factor in $\tilde{F}, i=0,1, \ldots n-1$. To construct one-factor $F_{m n+i}$ of $K_{p n+1}$, for $m=0,1, \ldots, p-1$ and $i=0,1, \ldots, n-1$, copies of all edges of $\tilde{F}_{i}$ are taken by replacing $\tilde{V}$ with $V_{m}$, and moreover, the set of edges $\left\{\left\{j_{m-s},-\left(j+(i+m) r^{-1}\right)_{m+s}\right\}: j=0,1, \ldots n-1, s=1,2, \ldots, r\right\}$ is added.

Lemma 14 For odd prime $p$ and for odd $n \geq p$ such that $\operatorname{gcd}(n,(p-1) / 2)=1$, and for even $k \geq 4$, where $k \neq \equiv, p+1 \quad(\bmod 2 p)$ or $k=p+1$, and moreover, $k / 2 \nmid n$, if there is a $k$-cycle free one-factorization of $K_{n+1}$, then a $k$-cycle free one-factorization of $K_{p n+1}$ exists.

Proof: Assume that a k-cycle free one-factorization $\tilde{F}$ of $K_{n+1}$ is given. Let $H$ be the union of two one-factors $F_{h}$ and $F_{i}$ in the one-factorization obtained by applying Construction E, where $h<i$ and $h, i \in\{0,1, \ldots, p n-1\}$.

Suppose that $h$ and $i$ satisfy $m n \leq h<i<(m+1) n$ for some $m \in\{0,1, \ldots, p-1\}$. Then $H$ does not contain a cycle of length $k$ with all vertices in $V_{m}$ because onefactorization induced by $V_{m}$ is the given $k$-cycle free one-factorization $\tilde{F}$ of $K_{n+1}$. Moreover, let $C_{l}$ be a cycle of $H$ with all vertices in $V \backslash V_{m}$ and let $y_{m-s}$ be a vertex of $C_{l}$, for some $s \in\{1,2 \ldots, r\}$. Note that $C_{l}$ is exactly the same cycle as in Case I of the proof of Lemma 11 and, $\operatorname{since} \operatorname{gcd}(k / 2, n)<k / 2$ by the assumption, $l \neq k$ is satisfied.

It remains to consider the case when $m n \leq h<(m+1) n$ and $q n \leq i<(q+1) n$ for some $m, q \in\{0,1, \ldots, p-1\}, m<q$. Let $z=q-m$. If $\infty$ is a vertex of a cycle $C_{l}$ in $H$, then $l \equiv p+1 \quad(\bmod 2 p)$, cf. Subcase II.A in the proof of Lemma 11. Moreover, neighbors of $\infty$ in $H$ are $h_{m}$ and $i_{q}$ and the first $p+1$ consecutive vertices along the cycle $C_{l}$ in $H$ (by Subcase II.A in the proof of Lemma 11) are: $\infty, i_{q}, \ldots,(h-z)_{m} \neq h_{m}$. Hence $l \neq p+1$. If $\infty$ is not a vertex of $C_{l}$ in $H$, then $l \equiv 0 \quad(\bmod 2 p)$, cf. Subcase II.B in the proof of Lemma 11. Thus $l \neq k$.

To prove main results one more construction, slightly different from Construction E, is needed.

Construction $\mathbf{F}$ Let $p \geq 3$ be a prime and $r=(p-1) / 2$. Let $n$ be an odd integer such that $n \geq p$ and $\operatorname{gcd}(n, r)=1$. Let $r^{-1}$ be the inverse of $r$ in $\mathbb{Z}_{n}$. In what follows, labels of vertices are taken modulo $n$ and moreover, indices are taken modulo $p$. Let $r=(p-1) / 2$. Let $V=V_{0} \cup V_{1} \cup \ldots \cup V_{p-1}$, where $V_{m}=\left\{\infty, 0_{m}, 1_{m}, \ldots,(n-1)_{m}\right\}$ for $m=0,1, \ldots, p-1$. Let $\tilde{F}$ be a $k$-cycle free one-factorization of $K_{n+1}$, where $\tilde{V}=$ $V\left(K_{n+1}\right)=\{\infty, 0,1, \ldots, n-1\}$. Let $\tilde{F}_{i}$ be a one-factor in $\tilde{F}, i=0,1, \ldots n-1$. To construct one-factor $F_{m n+i}$ of $K_{p n+1}$, for $m=0,1, \ldots, p-1$ and $i=0,1, \ldots, n-1$, copies of all edges of $\tilde{F}_{i}$ are taken by replacing $\tilde{V}$ with $V_{m}$, and the set of edges $\left\{\left\{j_{m-s},-\left(j+i r^{-1}\right)_{m+s}\right\}\right.$ : $j=0,1, \ldots n-1, s=1,2, \ldots, r\}$ is added. 
Lemma 15 For odd prime $p$ and for odd $n \geq 3$ such that $\operatorname{gcd}(n,(p-1) / 2)=1$, and for even $k \geq 4$ where $k \neq 2 p, k \neq p+1$ and moreover, $k / 2 \nmid n$, if there is a $k$-cycle free one-factorization of $K_{n+1}$, then a $k$-cycle free one-factorization of $K_{p n+1}$ exists.

Proof: Assume that a $k$-cycle free one-factorization $\tilde{F}$ of $K_{n}$ is given. Let $H$ be the union of two one-factors $F_{h}$ and $F_{i}$ in the one-factorization constructed according to Construction F, where $h<i$ and $h, i \in\{0,1, \ldots, p n-1\}$.

Suppose that $h$ and $i$ satisfy $m n \leq h<i<(m+1) n$ for some $m \in\{0,1, \ldots, p-1\}$. Then clearly $H$ does not contain a cycle of length $k$ with all vertices in $V_{m}$ because onefactorization induced by $V_{m}$ is the given one-factorization $\tilde{F}$ of $K_{n+1}$, which is $k$-cycle free. Let $C_{l}$ be a cycle of $H$ with all vertices in $V \backslash V_{m}$. In fact, all vertices of $C_{l}$ are in $V_{m-s} \cup V_{m+s}$ for some $s \in\{1,2 \ldots, r\}$. Clearly $l \leq 2 n$. Let $y_{m-s}$ be a vertex of $C_{l}$. Neighbors of the vertex $y_{m-s}$ in $H$ are $-\left(y+h r^{-1}\right)_{m+s}$ and $-\left(y+i r^{-1}\right)_{m+s}$. Consecutive vertices along the cycle $C_{l}$ are: $y_{m-s},-\left(y+h r^{-1}\right)_{m+s},\left(y+(h-i) r^{-1}\right)_{m-s}$, $-\left(y+(2 h-i) r^{-1}\right)_{m+s},\left(y+(2 h-2 i) r^{-1}\right)_{m-s}, \ldots,-\left(y+\frac{l h-(l-2) i}{2} r^{-1}\right)_{m+s}, y_{m-s}$, where $l$ is the minimum even positive integer such that $-y-\frac{l h-(l-2) i}{2} r^{-1} \equiv-y-i r^{-1} \quad(\bmod n)$. Since $0<(i-h) r^{-1}<n$, by the equivalence $\frac{l}{2}(i-h) r^{-1} \equiv 0 \quad(\bmod n)$ and Claim 1 , $\operatorname{gcd}\left(\frac{l}{2}, n\right)=l / 2$ holds. Thus $l \neq k$.

It remains to consider the case when $m n \leq h<(m+1) n$ and $q n \leq i<(q+1) n$ for some $m, q \in\{0,1, \ldots, p-1\}, m<q$. Let $z=q-m$. Assume that $\infty$ is a vertex of $C_{l}$ in $H$. Neighbors of $\infty$ in $H$ are $h_{m}$ and $i_{q}$. Note that $p+1$ consecutive vertices along $C_{l}$ in $H$ are: $\infty, i_{q}=i_{m+z},-(h+r i) r_{m-z}^{-1},(h+(r-1) i) r_{m+3 z}^{-1},-(2 h+(r-1) i) r_{m-3 z}^{-1},(2 h+(r-2) i) r_{m+5 z}^{-1}$, $\ldots,(r h+(r-r) i) r_{m+p z}^{-1}=h_{m}$. Hence $l=p+1 \neq k$. Consider the case when $\infty$ is not a vertex of $C_{l}$ in $H$. Let $(h+x)_{m}$ be a vertex of $C_{l}$ for some $x \neq 0$. Then neighbors of $(h+x)_{m}$ in $H$ are $(h-x)_{m}$ and $-\left(h+x+i r^{-1}\right)_{q+z}=-\left(h+x+i r^{-1}\right)_{m+2 z}$. Therefore, $2 p$ consecutive vertices along $C_{l}$ are: $(h+x)_{m},-(r x+r h+i) r_{m+2 z}^{-1},(r x+(r-1) h+i) r_{m-2 z}^{-1}$, $\ldots,(r x+(r-r) h+r i) r_{m-(p-1) z}^{-1}=(i+x)_{m+z},(i-x)_{m+z},-(-r x+h+r i) r_{m-z}^{-1}$, $(-r x+h+(r-1) i) r_{m+3 z}^{-1}, \ldots,(-r x+r h+(r-r) i) r_{m+p z}^{-1}=(h-x)_{m}$. Thus $l=2 p$ and, by the assumption, $l \neq k$.

Note that a one-factorization made by Construction $\mathrm{F}$ does not contain a cycle of length $n p+1$. Moreover, if $n=p$ and $G K_{n+1}$ is taken as a one-factorization $\tilde{F}$ of $K_{n+1}$, then one-factorization produced in this way is a known uniform one-factorization of $K_{p^{2}+1}$ with cycles of lengths $p+1,2 p, 2 p, \ldots 2 p$. Applying Construction $\mathrm{F}$ more than once for justobtained uniform one-factorization easily produces a series of uniform one-factorizations for all orders of the form $p^{x}+1, x \geq 2$, where every one-factor has one cycle of length $p+1$ and $\left(p^{x-1}-1\right) / 2$ cycles of length $2 p$, cf. [4].

\section{Main results}

The constructions presented in the previous section are used to prove general results on $k$-cycle free one-factorizations. 
Theorem 16 For each $n$ and each even $k \geq 4$ such that $k \neq 2 n$, the complete graph $K_{2 n}$ has a $k$-cycle free one-factorization.

Proof: Let $k=2^{\lambda_{0}} p_{1}^{\lambda_{1}} p_{2}^{\lambda_{2}} \ldots p_{w}^{\lambda_{w}}$ be the prime factorization of $k$ into non-trivial factors, $\lambda_{j} \geq 1$ for each $p_{j}$ and $p_{1}<p_{2}<\ldots<p_{w}$. Since $k$ is even, $\lambda_{0} \geq 1$. If $k>2 n$, by Claim 2 the assertion is true. Thus, the result is trivial for $n=4$. In what follows, let $k<2 n$. For the induction, assume that a $k$-free one-factorization of $K_{2 m}$ exists for every $m$ such that $2 \leq m<n$ and $2 m \neq k$. Consider separately two cases:

Case I: $k / 2 \nmid n$. Thus $k \neq n$. For odd $n$, by Corollary 7 , the one-factorization $G A_{2 n}$ is $k$-cycle free. Assume that $n$ is even. If $\lambda_{0} \neq 2$, then to find a required one-factorization of $K_{2 n}$ apply Lemma 9. Consider the case $\lambda_{0}=2$. Note that $k>4$ because otherwise $k / 2=2 \mid n$. Let $x=\max \left\{y: \operatorname{gcd}\left(2^{y}, n\right)=2^{y}\right\}$. Hence immediately $k \neq n / 2^{y}$ for every $y \leq x$. Let $n^{\prime}=n / 2^{x}$. Note that both $k / 2 \nmid n^{\prime}$ and $k / 4 \nmid n^{\prime}$. Thus, the one-factorization $G A_{2 n^{\prime}}$ of $K_{2 n^{\prime}}$, by Corollary 7 , is $\{k / 2, k\}$-cycle free. In the next steps apply $x$ times Construction $\mathrm{C}$ to get, by Lemma 10, one-factorizations of $K_{4 n^{\prime}}$, of $K_{8 n^{\prime}}, \ldots$, of $K_{2 n}$, respectively, which are $\{k / 2, k\}$-cycle free.

Case II: $k / 2 \mid n$. Hence, for every $j=1,2, \ldots, w, p_{j} \mid n$ and clearly $p_{j} \nmid 2 n-1$. Thus $\operatorname{gcd}(k / 2,2 n-1)=1$. If $\operatorname{gcd}(k-1,2 n-1)<k-1$, by Corollary 4 the one-factorization $G K_{2 n}$ is $k$-cycle free. Consider the opposite case $\operatorname{gcd}(k-1,2 n-1)=k-1$. Let $f$ be the minimum nontrivial factor of $2 n-1$ and $e=\frac{2 n-1}{f}$. Thus $e \geq f \geq 3$ and $\operatorname{gcd}(e,(f-1) / 2)=1$. Moreover, since $\operatorname{gcd}(k / 2, e f)=1, \operatorname{gcd}(k / 2, e)=1$ and $f \nmid k / 2$ immediately follow, and then $k \neq \equiv 0 \quad(\bmod 2 f)$. The aim is to show that $e \neq k-1$. Suppose to the contrary that $e=k-1$. Then $2 n-1=e f=(k-1) f$ and, since $n=z \frac{k}{2}$ for some integer $z$, $k(f-z)=f-1$. Thus, $k$ is a divisor of $f-1$, whence $f \geq k+1=e+2$, which contradicts the fact that $f$ is the minimum factor of $2 n-1$. By the inductive assumption there is a $k$-cycle free one-factorization of $K_{e+1}$. If $f$ is not a factor of $k-1$ (it means $k \not \equiv f+1$ $(\bmod 2 f))$ or $f=k-1$, then to find a required one-factorization of $K_{e f+1}$ apply Lemma 14 (with $p:=f$ ). Otherwise $f \mid k-1$ and $f<k-1$. In this case, to find a $k$-cycle free one-factorization of $K_{e f+1}$, apply Lemma 15 (with $p:=f$ ).

The existence of 4-cycle free one-factorizations of complete graphs has already been stated in [9].

For an infinite class of even orders $2 n$ of complete graphs, $2 n$-cycle free one-factorizations may be constructed. Note that all one-factorizations $G K_{2 n}, G A_{2 n}$, as well as $H K_{2 n}$, are not useful for this purpose since, as was noted earlier, they contain Hamiltonian cycles.

Theorem 17 Let $2 n \neq p+1$, where $p$ is a prime, or $2 n \not \equiv 6,12,18$ (mod 24). Then the complete graph $K_{2 n}$ has a $2 n$-cycle free one-factorization.

Proof: Let $2 n \neq p+1$ for every prime $p$. Let $f$ be the minimum prime factor of $2 n-1$ and $e=\frac{2 n-1}{f}$. Then $e \geq f \geq 3$ and to construct a $2 n$-cycle free one-factorization of $K_{e f+1}$ apply, by Lemma 15, Construction F.

If $2 n \equiv 2,4 \quad(\bmod 6)$, then it is easily observed than any Steiner one-factorization of order $2 n$ (cf. [12]) is $2 n$-cycle free; in fact, the union of any two one-factors contains 
the cycle $C_{4}$. If $2 n \equiv 0 \quad(\bmod 8)$, then $n$ is even and, by Claim 2, any one-factorization of $K_{n}$ is $2 n$-cycle free. Hence, by Lemma 9, Construction $\mathrm{C}$ produces a required onefactorization.

At present, the existence problem of $k$-cycle free one-factorizations when $k=2 n$ has been only partially solved. In contrast to perfect one-factorizations, orders of the form $2 n=p+1$, for $p$ being prime, appear to be the most difficult regarding constructions of $2 n$-cycle free one-factorizations of $K_{2 n}$. However, the existence of $n$-cycle free onefactorization of $K_{n}$ when $n \equiv 2 \quad(\bmod 4)$, by Lemma 10 , immediately implies the existence of $2 n$-cycle free one-factorization of $K_{2 n}$. Moreover, known examples of non-perfect uniform one-factorizations of $K_{2 n}$ (cf. [5]), as well as the $2 n$-cycle free one-factorizations for $2 n=18$ given in the Appendix, cover all unsolved cases for orders less than 102 .

The more general question concerns $k^{<}$-cycle free one-factorizations of the complete graph. This appears to be much more difficult. One obvious argument is that perfect one-factorizations of $K_{2 n}$ are simply $(2\lfloor n / 2\rfloor)^{<}$-cycle free one-factorizations. Even for $k=6$, all constructions presented in this paper are not sufficient to obtain a complete classification, i.e. the case $2 n=28$ remains unsolved. However, for every order $2 n \equiv 2$ $(\bmod 4)$, a $6^{<}$-cycle free one-factorization of $K_{2 n}$ may be constructed.

Theorem 18 For every odd $n \geq 5$, there exists one-factorization of $K_{2 n}$ which is $6^{<}$-cycle free.

Proof: Let $q$ be the minimum prime factor of $n$. If $q \geq 5$, then the one-factorization $G A_{2 n}$, by Corollary 8, is $8^{<}$-cycle free. Therefore, assume that $q=3$. Clearly, $3 \nmid 2 n-1$. If 5 is not a factor of $2 n-1$, then the one-factorization $G K_{2 n}$, by Corollary 5 , is $6^{<}$-cycle free. It remains to consider the case when $5 \mid 2 n-1$. Let $2 n-1=r_{1} r_{2} \ldots r_{v}$ be the prime factorization of $2 n-1$ into non-trivial factors, where $5=r_{1} \leq r_{2} \leq \ldots \leq r_{v}$ and $v \geq 2$. Note that for $r_{v} \geq 7$ there exists a $6^{<}$-cycle free one-factorization $\hat{F}$ of $K_{r_{v}+1}$, namely, by Corollary 5 , as $\hat{F}$ the one-factorization $G K_{v+1}$ may be substituted. Otherwise $r_{v}=5$ and $2 n-1=5^{x}$ for some $x \geq 2$. Let $\hat{F}$ be the one-factorization $G A_{5^{2}+1}$ of $K_{5^{2}+1}$ which is clearly perfect. In the next steps apply $v-1$ times $\left(v-2\right.$ times if $\left.r_{v}=5\right)$ the inductive Construction E, taking as $p$ 's consecutive prime factors of $2 n-1$ in the non-increasing order. In this way, by Lemma 14 , a series of $6^{<}$-cycle free one-factorizations is constructed, ending up at the order $2 n$.

Although it is not possible to construct $k^{<}$-cycle free one-factorizations for all orders $2 n \geq k \geq 6$, infinite families of orders may be provided, for which such one-factorizations do exist. Evidently, by Corollary 5, the one-factorization $G K_{2 n}$ is $k^{<}$-cycle free for every order $2 n$ such that the prime factorization of $2 n-1$ does not contain a factor less than $k$. Let $n \geq 3$ and let $r$ be the minimum prime factor of $2 n-1$. Moreover, let $l=$ $\max \left\{r_{1}-1,2 r_{2}-2\right\}$, where $r_{1}$ is the minimum prime factor of $\frac{2 n-1}{r}$ and $r_{2}$ the minimum prime factor of $n$. If $r \geq 5$, then there exists an $l^{<}$-cycle free one-factorization of $K_{2 n}$ (which follows directly from Corollaries 8 and 13). 
Acknowledgement The research was supported by the Foundation for Polish Science Grant for Young Scholars.

\section{References}

[1] B.A. Anderson, Finite topologies and Hamiltonian paths, J. Combin. Theory Ser. B 14 (1973) 87-93.

[2] B.A. Anderson, Symmetry groups of some perfect one-factorizations of complete graphs, Discrete Math. 18 (1977) 227-234.

[3] D. Bryant, B. Maenhaut, I.M. Wanless, New families of atomic Latin squares and perfect 1-factorizations, J. Combin. Theory Ser. A 113 (2006) 608-624.

[4] P.J. Cameron, Parallelisms of complete designs, London Mathematical Society Lecture Note Series No. 23 Cambridge University Press, Cambridge 1976.

[5] J.H. Dinitz, P. Dukes, On the structure of uniform one-factorizations from starters in finite fields, Finite Fields Appl. 12 (2006) 283-300.

[6] J.H. Dinitz, P. Dukes, D.R. Stinson, Sequentially perfect and uniform one-factorizations of the complete graph, Electron. J. Combin. 12 (2005) \# R1 12 pp.

[7] J.H. Dinitz, D.K. Garnick, There are 23 nonisomorphic perfect one-factorizations of $K_{14}$, J. Combin. Des. 4 (1996) 1-4.

[8] J.H. Dinitz, D.K. Garnick, B.D. McKay, There are 526, 915, 620 nonisomorphic onefactorizations of $K_{12}$, J. Combin. Des. 2 (1994) 273-285.

[9] D. Fronček, A. Rosa, Symmetric graph designs on friendship graphs, J. Combin. Des. 8 (2000) 201-206.

[10] P. Kaski, P.R.J. Östergård, There are 1, 132, 835, 421, 602, 062, 347 nonisomorphic one-factorizations of $K_{14}$, J. Combin. Des., in print.

[11] A. Kotzig, Hamilton graphs and Hamilton circuits, Theory of Graphs and Its Applications, Proc. Sympos. Smolenice 1963, Nakl. CSAV 1964, pp. 63-82.

[12] C.C. Lindner, E. Mendelsohn, A. Rosa, On the number of 1-factorizations of the complete graph, J. Combin. Theory Ser. B 20 (1976) 265-282.

[13] E.Lucas, Récréations Mathématiques vol. II, Gauthier-Villars, Paris 1883.

[14] E. Mendelsohn, A. Rosa, One-factorizations of the complete graph-a survey, J. Graph Theory 9 (1985) 43-65.

[15] M. Meszka, A. Rosa, Perfect 1-factorizations of $K_{16}$ with nontrivial automorphism group, J. Combin. Math. Combin. Comput. 47 (2003) 97-111.

[16] E. Seah, Perfect one-factorizations of the complete graph-a survey, Bull. Inst. Combin. Appl. 1 (1991) 59-70.

[17] W.D. Wallis, One-factorizations of complete graphs, Contemporary Design Theory: A Collection of Surveys (Ed. J.H. Dinitz, D.R. Stinson), Wiley 1992, pp. 593-631. 


\section{Appendix}

One-factors of 18-cycle free one-factorization of $K_{18}, V\left(K_{18}\right)=\{0,1, \ldots, 17\}$ :

0-1,2-3,4-5,6-7,8-17,9-10,11-12,13-14,15-16; 0-2,1-3,4-7,5-8,6-15,9-11,10-12,13-16,14-17;

0-3,1-2,4-13,5-6,7-8,9-12,10-11,14-15,16-17; $\quad 0-4,1-5,2-6,3-8,7-16,9-13,10-14,11-15,12-17$;

0-5,1-4,2-11,3-7,6-8,9-14,10-13,12-16,15-17; $0-6,1-7,2-8,3-4,5-14,9-15,10-16,11-17,12-13$;

0-7,1-6,2-5,3-12,4-8,9-16,10-15,11-14,13-17; $0-8,1-10,2-4,3-6,5-7,9-17,11-13,12-15,14-16$;

0-9,1-8,2-7,3-5,4-6,10-17,11-16,12-14,13-15; $0-10,1-9,2-12,3-11,4-14,5-16,6-17,7-15,8-13$;

0-11,1-17,2-16,3-9,4-15,5-12,6-13,7-14,8-10; $0-12,1-11,2-10,3-15,4-9,5-13,6-14,7-17,8-16$;

0-13,1-14,2-15,3-17,4-16,5-10,6-11,7-12,8-9; $0-14,1-13,2-17,3-16,4-10,5-9,6-12,7-11,8-15$;

0-15,1-12,2-9,3-10,4-11,5-17,6-16,7-13,8-14; 0-16,1-15,2-13,3-14,4-17,5-11,6-10,7-9,8-12;

0-17,1-16,2-14,3-13,4-12,5-15,6-9,7-10,8-11. 